\title{
Redox-regulated molecular chaperones
}

\author{
P. C. F. Graf ${ }^{\mathrm{b}}$ and U. Jakob ${ }^{\mathrm{a}, \mathrm{b}, *}$ \\ a Department of Molecular, Cellular and Developmental Biology, University of Michigan, 830 N. University Avenue, \\ Ann Arbor, Michigan 48109-1048 (USA), Fax +1 734647 0884, e-mail: ujakob@umich.edu \\ ${ }^{\mathrm{b}}$ Program in Cellular and Molecular Biology, University of Michigan, Ann Arbor, Michigan 48109-1048 (USA)
}

\begin{abstract}
The conserved heat shock protein Hsp33 functions as a potent molecular chaperone with a highly sophisticated regulation. On transcriptional level, the Hsp33 gene is under heat shock control; on posttranslational level, the Hsp33 protein is under oxidative stress control. This dual regulation appears to reflect the close but rather neglected connection between heat shock and oxidative stress. The redox sensor in Hsp33 is a cysteine center that coordinates zinc under reducing, inactivating conditions and that forms two intramolecular disulfide
\end{abstract}

bonds under oxidizing, activating conditions. Hsp33's redox-regulated chaperone activity appears to specifically protect proteins and cells from the otherwise deleterious effects of reactive oxygen species. That redox regulation of chaperone activity is not restricted to Hsp33 became evident when the chaperone activity of protein disulfide isomerase was recently also shown to cycle between a low- and high-affinity substrate binding state, depending on the redox state of its cysteines.

Key words. Hsp33; PDI; redox regulation; disulfide bond formation; oxidative stress; heat shock; metal centers; reactive oxygen species.

\section{Hsp33-A chaperone on the interface of oxidative stress and heat shock}

Hsp33 is a highly conserved heat shock protein that is present in the cytosol of more than 50 different prokaryotic organisms. Recently, the first eukaryotic Hsp33 homologue was identified in the unicellular photosynthetic organism Chlamydomonas reinhardtii, where it is predicted to be localized to the chloroplasts by PSORT [1].

The transcription of $h s l O$ (originally named for heat shock locus $\mathrm{O}$ ), the gene encoding Hsp33, is under heat shock control [2]. In Escherichia coli, hslO forms part of a heat-shock-regulated multigene operon. It is located downstream of $h s l R$, a gene encoding the $50 \mathrm{~S}$ ribosomal binding protein Hsp15 [3], and $h s l P$, a gene encoding a putative heat-shock-regulated phosphatase. Upon exposure of $E$. coli to heat shock conditions, the messenger RNA (mRNA) level of the Hsp33 gene increases about 30 -fold, while the mRNA level of $h s l R$ and $h s l P$ increase about 40- and 20-fold, respectively [4]. This increases the steady-state protein concentration of Hsp33 to $\sim 3 \mu \mathrm{M}$,

\footnotetext{
* Corresponding author.
}

twice the level of Hsp33 under nonstress conditions [5]. This is similar to the concentration reported for the double-doughnut chaperonin GroEL $(2.6 \mu \mathrm{M})$ [6].

Thus, on transcriptional level, the Hsp33 gene is under heat shock control; on posttranslational level, the Hsp33 protein is under oxidative stress control [5]. This posttranslational mode of regulation enables the Hsp33 protein to sense changes in the redox conditions of the environment and to translate these changes into differences in protein conformation and chaperone activity. Hsp33's redox sensor is located in the C-terminus of the protein. It consists of four highly conserved cysteines that coordinate zinc in the reduced, low-affinity substrate-binding conformation, and that form two intramolecular disulfide bonds in the oxidized, high-affinity substrate-binding conformation of Hsp33 [5]. The redox potential of Hsp 33 's chaperone activity has been shown to be $-170 \mathrm{mV}$ [5]. This implies that under nonstress conditions, where the bacterial cytosol is thought to be strongly reducing $(-250$ to $-280 \mathrm{mV})$ [7], Hsp33's cysteines coordinate zinc, and the protein is mostly deactivated. Under oxidative stress conditions, on the other hand, where the cellular redox potential can increase to values of $-150 \mathrm{mV}$ 
or more [7], Hsp33's cysteines become oxidized, and the protein is activated [5] (also reviewed by $[8,9]$ ). The redox potential of Hsp33 is very similar to the redox potential of OxyR, the oxidative stress transcription factor in E. coli $(-185 \mathrm{mV})$ [10]. OxyR is preferentially reduced and inactive under normal conditions, but becomes rapidly activated upon exposure of the cells to $\mathrm{H}_{2} \mathrm{O}_{2}$ [10-12]. Similar to Hsp33, OxyR's activation mechanism involves the formation of an intramolecular disulfide bond. Noteworthy, Hsp33's transcription is not under OxyR control [13, 14].

Hsp33's activation on a posttranslational level under oxidative stress will lead to a certain level of chaperone activity that might be sufficient for the cells as a first line of defense against agents that cause oxidative damage. When the oxidative stress conditions lead to such severe protein damage that the heat shock response is induced, Hsp33's concentration will also increase.

\section{The on-off switch: a cysteine coordinating zinc center}

Hsp33 from E. coli has six cysteine residues, four of which are highly conserved. These four conserved cysteines, Cys-X-Cys- $\mathrm{X}_{27-32}$-Cys-X-X-Cys, form a novel, very high affinity zinc binding motif [15]. The $K_{\mathrm{a}}$ of reduced $\mathrm{Hsp} 33$ for zinc(II) at $25^{\circ} \mathrm{C}, \mathrm{pH} 7.5$ is $2.5 \times 10^{17} \mathrm{M}^{-1}$ [15]. This indicates a stronger interaction between zinc and Hsp33 than between zinc and the cytosolic zinc storage protein, metallothionein, which has a $K_{\mathrm{a}}$ of $3.2 \times$ $10^{13} \mathrm{M}^{-1}, \mathrm{pH} 7.4[16,17]$ and many other zinc-binding proteins. The zinc ion appears to be coordinated by all four conserved cysteines in a tetrahedral geometry, as evidenced by metal replacement studies with cobalt(II) [15]. The zinc center in Hsp33 folds in a very stable domain that is highly protease resistant when compared with zinc-free reduced Hsp33 [15]. Not even the unfolding of Hsp33 in $6 \mathrm{M}$ guanidine hydrochloride causes the release of the bound zinc [15]. In contrast, exposure of Hsp33 to oxidizing conditions (e.g. $\mathrm{H}_{2} \mathrm{O}_{2}$ ) releases the zinc immediately. This appears to be due to the formation of two intramolecular disulfide bonds, which connect the two next-neighbor cysteines $\mathrm{Cys}_{232}$ with $\mathrm{Cys}_{234}$ and $\mathrm{Cys}_{265}$ with $\mathrm{Cys}_{268}$ [18]. This oxidation induced disulfide bond formation and zinc release is the first step in the activation process of Hsp33 [19].

Despite the fact that zinc is a redox inert metal, experiments with zinc-reconstituted and zinc-free reduced Hsp33 have shown that zinc plays a rather active role in the activation process of Hsp33. Zinc-free Hsp33 reactivates slowly and incompletely when exposed to oxidative stress treatment in vitro, whereas the reactivation of zinccoordinated Hsp33 occurs quickly and goes to completion [15]. We have shown that all four cysteines of Hsp33 are in their highly reactive thiolate anion state when the metal center is occupied. This may be due to the zinc(II) cation acting as a Lewis acid that can stabilize the negative charges on the thiolate anions, thereby lowering their $\mathrm{p} K_{\mathrm{a}}$ values and making them more reactive to hydrogen peroxide at near neutral $\mathrm{pH}[20]$. This thiolate anion state in combination with the close proximity of the thiol groups in the tertiary structure has been postulated to prime the cysteines for a rapid reaction to form disulfide bonds [15].

\section{Activation: a two-step process}

Biochemical studies revealed that Hsp33's activation process occurs in two steps [19]. The first step involves formation of two intramolecular disulfide bonds and concurrent release of zinc. This step is independent of Hsp33 protein concentration and occurs prior to the full activation of Hsp33, a process that involves dimerization of two oxidized Hsp33 monomers. This second step is both highly temperature and concentration dependent, optimal for a protein that is overexpressed under heat shock conditions. Oxidized and fully active Hsp33 dimer has a $K_{\mathrm{D}}$ of $0.6 \mu \mathrm{M}$ at $20^{\circ} \mathrm{C}$ [19]. Since the dimerization of oxidized Hsp33 appears to be dependent on hydrophobic interactions between both monomers at the dimer interface [19], elevated temperatures should lower the $K_{\mathrm{D}}$, favor the dimerization process and significantly accelerate the activation of Hsp33 under heat shock conditions. Gel filtration studies with a constitutively active Hsp33 truncation mutant (aa 1-235) confirmed the presence of dimers at physiological Hsp33 concentrations. At lower Hsp33 concentrations, addition of denatured protein substrates has been shown to shift the equilibrium of the oxidized truncation mutant toward the dimeric conformation [21].

Extensive conformational changes appear to accompany the activation process of Hsp33. Far ultraviolet (UV)-circular dichroism spectra revealed that the oxidation of reduced Hsp33 is accompanied by significant changes in its secondary structure. Based on this analysis, reduced Hsp33 is predicted to contain $\sim 31 \%$ random coil, $44 \% \alpha$ helix and $25 \% \beta$ sheet, while the oxidized, active Hsp33 revealed significantly increased random coil $(49 \%)$ at the expense of $\alpha$ helix (35\%) [22]. A substantial increase in hydrophobic surfaces has been shown to accompany the activation process of Hsp33 [22]. This may be important in Hsp33's chaperone function. Using the fluorescence properties of the small hydrophobic dye bis-ANS, it has been demonstrated that while reduced Hsp33 barely interacts with the hydrophobic probe, oxidized Hsp33 shows a major blue-shifted fluorescence signal [22]. This suggests that substantial rearrangements occur upon oxidation and dimerization of Hsp33 that allow the formation or exposure of a predominantly hydrophobic sub- 
strate binding site that is either nonexistent or buried in the reduced, inactive Hsp33 monomer.

\section{Hsp33's substrates}

In vitro, oxidized Hsp33 is a very potent molecular chaperone. Substoichiometric amounts of active Hsp33 are sufficient to completely prevent the thermal aggregation of two classical in vitro chaperone substrates, firefly luciferase and porcine mitochondria citrate synthase [5], as well as of the eye lens protein $\zeta$-crystallin [22]. For chemically denatured luciferase and citrate synthase, active Hsp33 dimers need to be present in at least 2:1 molar ratios to effectively prevent aggregation $[18,19][\mathrm{J}$. H. Hoffmann et al., unpublished observations]. Oxidized Hsp33 is also able to interact with chemically denatured rhodanese [P. C. F. Graf and U. Jakob, unpublished observations] and has been shown to efficiently prevent the aggregation of oxidatively damaged RrmJ (FtsJ), a methyltransferase of $E$. coli [5]. The stoichiometry with which active Hsp33 dimers are capable of preventing the aggregation of folding intermediates that derive from either chemically or thermally denatured proteins makes Hsp33 fivefold more effective on a mass ratio basis than GroEL [5]. In contrast, the activity of reduced zinc-oordinated Hsp33 is very substantially downregulated. In activity assays where the influence of Hsp33 on chemically denatured substrates is tested, not even a 10 -fold molar excess of reduced Hsp33 reveals any substantial influence on the aggregation behavior of either luciferase or citrate synthase [19] [J. H. Hoffmann et al., unpublished observations]. Using thermally unfolding protein intermediates as substrates, reduced Hsp33 shows a very marginal, $<10 \%$ influence on the aggregation process as compared with oxidized active Hsp33 [5, 22].

From all the substrate proteins that have been tested with E. coli Hsp33 in vitro, only RrmJ (FtsJ) is an E. coli protein. All others are heterologous proteins; luciferase, for instance, is isolated from firefly, while citrate synthase is purified from porcine heart mitochondria. However, these are well-characterized chaperone substrates and therefore well suited to analyze the mechanism of Hsp33's chaperone action. We are now in the process of identifying the in vivo substrates of Hsp33. Preliminary data indicate that Hsp33 is capable of suppressing the aggregation of a variety of $E$. coli proteins ranging in size from 10 to $200 \mathrm{kDa}$ [U. Jakob, unpublished results].

\section{Hsp33: a holdase}

Oxidized, active Hsp33 appears to act as protein 'holdase' in vitro. It efficiently prevents the aggregation of folding intermediates but seems unable to mediate the productive refolding of the substrate proteins, once the environmental conditions return to normal. For instance, reactivation of a substantial amount of thermally inactivated citrate synthase can be induced by the addition of its substrate oxaloacetic acid (OAA) [23, 24]. In the presence of active Hsp33, however, OAA-induced refolding and reactivation of thermally denatured citrate synthase is negligible [5]. This can be explained in several ways: (i) Hsp33 binds very tightly to the folding intermediates of citrate synthase, and folding intermediates released from Hsp33 rebind to Hsp33 much faster than they can refold. This would be similar to the tight binding of unfolding intermediates to GroEL, observed in the absence of GroES and ATP [24]. To prevent rebinding and allowing effective refolding of the substrate protein, addition of a yetto-be-defined thiol-reducing agent or protein cofactor might be necessary. (ii) Hsp33 binding to unfolding intermediates causes the further unfolding of the folding intermediates. This would shift the equilibrium further toward the unfolded state and prevent the refolding of the protein.

The high affinity with which Hsp33 binds its substrate proteins provides a very plausible explanation as to why Hsp33's activity is so tightly regulated. Hsp33's chaperone activity might need to be downregulated under nonstress conditions to prevent Hsp33 from interacting with protein-folding intermediates that are on the folding pathway and, therefore, from interfering with the normal protein-folding process.

\section{The Hsp33 structure: a novel fold}

The structure of the active Hsp33 dimer has been independently solved by two groups [21,25]. Structure analysis revealed that Hsp33 has a novel protein fold. The Nterminal 178 amino acids fold into a compact core domain where two antiparallel $\beta$ sheets consisting of five and four $\beta$ strands, respectively, and two short helices ( $\alpha 1$ and $\alpha 4$ ) form a flattened barrel around a 18 -amino acid long central $\alpha$ helix ( $\alpha 2)$. One topologically similar protein to this domain is green fluorescent protein from Aequorea $(\mathrm{Z}$ score $=5.5)[25]$ that also forms a barrel around a buried $\alpha$ helix. In green fluorescent protein, however, the helix is completely surrounded by antiparallel $\beta$ strands.

After a highly conserved proline residue (P178), the Hsp33 polypeptide crosses to the other subunit and forms a domain swapped dimer (fig. 1). As with so many domain-swapped molecules, it is unclear whether this domain swapping in Hsp33 also occurs in solution. Both structures lacked the C-terminal zinc binding region (aa 234-294 and 236-294, respectively), and mass spectrometric analysis revealed that proteolytic cleavage occurred at residue 255 during the crystallization process of 


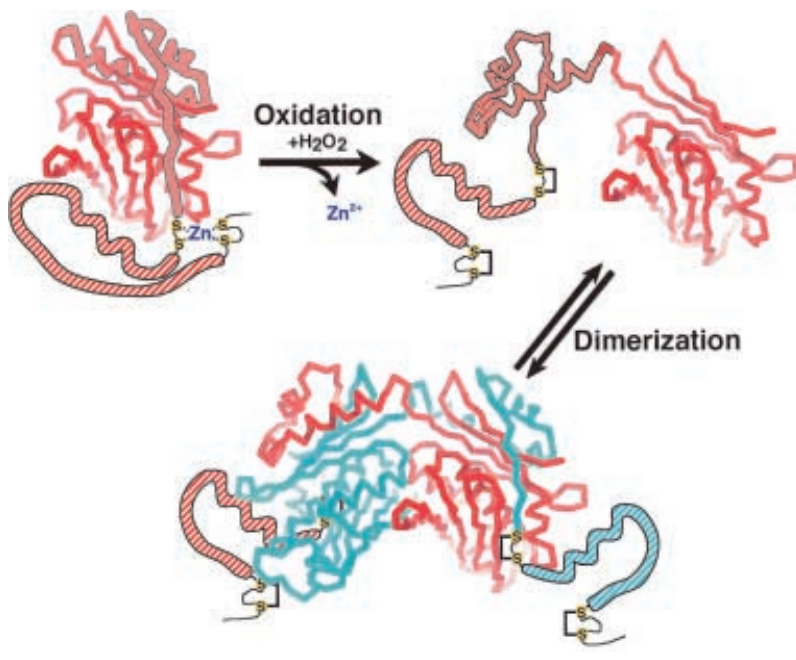

Figure 1. Schematic model of Hsp33 dimerization and activation derived from structural and solution results $[19,25]$. Zinc-reconstituted, reduced Hsp33 forms a stable monomer in solution and is inactive as a molecular chaperone. The zinc-binding carboxy-terminal domain of Hsp33 is postulated to block the substrate binding site and/or the dimerization interface. Upon incubation in $\mathrm{H}_{2} \mathrm{O}_{2}$, two intramolecular disulfide bonds form and zinc is released. In a subsequent concentration-dependent process, two oxidized monomers associate and form a highly active Hsp33 dimer. The four conserved cysteines that ligate zinc in reduced Hsp33 and form disulfide bonds in the oxidized form are shown in yellow. The modeled regions are drawn as thick hatched lines. The solid tubes show the Hsp33 structure as solved by Vijayalakshmi et al. [25, 57].

Hsp33 [25]. In addition, electron density for residues 236-255 could not be resolved, indicating that amino acids 235-255 are very flexible.

Analysis of the Hsp33 surface revealed the presence of two potential substrate binding sites in the Hsp33 dimer, a saddle-shaped $\beta$ sheet that presents $2250 \AA$ of accessible surface $[21,25]$ and an elongated groove that runs across the subunits with a length of $\sim 50 \AA$ and a width of $6-8 \AA$ [25]. Both putative substrate binding sites are characterized by an assembly of hydrophobic niches that should be able to accommodate hydrophobic residues that are exposed as Hsp33's substrates begin to unfold, as well as conserved patches of polar residues that could interact with the peptide backbone of the substrate proteins. The remaining surface of Hsp33 is highly acidic, presumably insufficient to interact with unfolding polypeptides.

\section{Regulation of Hsp33: a model}

Analysis of Hsp33's crystal structure in combination with the biochemical studies led to a model which describes how changes in the redox conditions might lead to the activation of Hsp33's molecular chaperone function (fig.1) [19]. Under reducing conditions, Hsp33 is monomeric, and all four cysteines are involved in zinc coordination. In this conformation, Hsp33's surface is largely nonhy- drophobic [22], and the protein lacks the ability to interact with folding intermediates. The function of the C-terminal zinc binding domain might either be to prevent the dimerization process by masking the dimer interface, or to interact and occupy the substrate binding site [21, 25]. Disulfide bond formation and the simultaneous structural rearrangement is proposed to lead to the displacement of the C-terminus, a process that favors dimerization and causes the formation or exposure of Hsp33's substrate binding site.

\section{The 'Minuteman' Hsp33: a defense strategy against disulfide stress}

Oxidative stress is a stress situation that is simply unavoidable for organisms that live an aerobic lifestyle. Autooxidation of components of the respiratory chain leads to the generation of superoxide anions and $\mathrm{H}_{2} \mathrm{O}_{2}$ under normal conditions [26]. Therefore, various detoxifying enzymes such as superoxide dismutase (SOD) and catalase have evolved that deal directly with the reactive oxygen species (ROS). Moreover, the E. coli cytoplasm is kept in a highly reducing environment by the action of two protein thiol-disulfide reducing systems, the thioredoxin and glutathione pathways (for review see [27]). These systems prevent the formation of intracellular disulfide bonds, a process that would lead to the inactivation of many cytosolic proteins [7].

These strategies of general oxidative defense have, however, been found to be inadequate for cells that face increasing concentrations of reactive oxygen species, such as can occur during heat shock [28] or oxidative attack by phagocytes [26, 29]. To kill invading bacteria, phagocytes release high concentrations of superoxide anions, which are converted into hydrogen peroxide by SOD [30]. At the same time, superoxide anions also rapidly reduce oxidized transition metals such as $\mathrm{Fe}(\mathrm{III})$ and $\mathrm{Cu}(\mathrm{II})$, yielding diatomic molecular oxygen and $\mathrm{Fe}(\mathrm{II})$ or $\mathrm{Cu}(\mathrm{I})$ [31]. These reduced metals are then free to react with hydrogen peroxide via the Fenton reaction to create very reactive hydroxyl radicals $(\mathrm{OH} \bullet)$ [31]. $\mathrm{H}_{2} \mathrm{O}_{2}$ and hydroxyl radicals can then lead to irreversible protein damage, side-chain oxidation, nonspecific disulfide bond formation, thermal instability, aggregation and finally cell death [7, 32].

We have observed that Hsp33's chaperone activity is rapidly turned on by hydroxyl radicals in vitro [15]. If this is also the case in vivo, then the activation of Hsp33 should parallel nonspecific and damaging disulfide bond formation in cytosolic proteins and deletion of Hsp33 should make cells more susceptible to oxidative protein damage. In vivo trapping experiments using the thiol-specific trapping agent 4-acetamido-4'-maleimidylstilbene2,2'-disulfonate (AMS) revealed that this is likely to be the case [5]. Under reducing conditions, Hsp33 is pre- 
dominantly reduced and presumably inactive. Strains that are known to accumulate disulfide-bonded proteins in the cytoplasm due to the absence of the thioredoxin and glutathione system, on the other hand, accumulate up to $60 \%$ of Hsp33 in the disulfide-bonded, active conformation [5]. These cells show a significantly higher resistance to $\mathrm{H}_{2} \mathrm{O}_{2}$ treatment, which appears to be in part due to the presence of oxidized, active Hsp33. Deletion of the Hsp33 gene in these constitutively oxidatively stressed mutant cells leads to a 10,000-fold increase in $\mathrm{H}_{2} \mathrm{O}_{2}$ sensitivity [5]. We therefore believe that activation of Hsp33's chaperone function represents a first line of defense strategy against oxidative protein damage. Conditions that lead to nonspecific disulfide bond formation in cytosolic proteins seem at the same time to lead to activation of Hsp33's chaperone function. Activated Hsp33 is then able to bind these nonnative proteins and prevent aggregation processes. Keeping Hsp33 in an inactive, yet 'ready-to-go' conformation in the cell under normal growth conditions is a very clever way to quickly respond to those sudden environmental changes.

\section{Heat shock and oxidative stress: two sides of the same coin?}

Heat shock and oxidative stress response are two closely connected stress responses in the cell. It has been long known that heat-shock-treated Chinese hamster fibroblasts are more resistant to subsequent hydrogen peroxide $\left(\mathrm{H}_{2} \mathrm{O}_{2}\right)$ treatment [33]. This is most likely due to the expression of heat shock proteins that protect the cell against the lethal consequences of oxidative stress. On the other hand, oxidative stress has been shown to play a major role in heatshock-induced cell death in yeast, possibly because many cellular antioxidant proteins are thermally unstable [34-36]. Deletion of antioxidant proteins such as SOD or catalase makes strains more sensitive to the lethal effects of heat, while overexpression of these enzymes significantly increases their thermotolerance [34, 35]. Moreover, growth of yeast cells in the absence of oxygen causes a 500- to 20,000-fold increase in thermotolerance [34]. Two-dimensional gel electrophoresis in eukaryotes and prokaryotes revealed that heat shock induces the expression of certain antioxidant proteins [37], while oxidative stress was shown to induce a subset of heat shock proteins [32, 37-39]. The cross talk observed between these two cellular stress responses might be based on the deleterious effect of reactive oxygen species on proteins under oxidative stress conditions that causes their unfolding and aggregation [40] and, therefore, induction of the heat shock response, and the thermal instability of certain antioxidant proteins under heat shock conditions, which causes accumulation of reactive oxygen species and induction of the oxidative stress response [32].

\section{PDI: a second redox-regulated chaperone}

Protein disulfide isomerase (PDI) is a multifunctional eukaryotic enzyme that contains four thioredoxin-like folds. Two of these four thioredoxin-like domains have redox active CXXC motifs. PDI functions as an oxidoreductase, isomerase and ATP-independent molecular chaperone and has been found to be localized to the rather oxidizing environment of the endoplasmic reticulum (ER) (for review see [41]). When PDI acts as an oxidase to form disulfide bonds in newly translocated ER proteins, PDI's cysteines are oxidized. When PDI acts as isomerase to reshuffle wrongly formed disulfide bonds in ER proteins, PDI's cysteines are reduced (for review see [41]).

Recently, Tsai and co-workers proposed that PDI acts as a redox-regulated chaperone, able to disassemble cholera toxin in a redox-dependent manner [42]. Reduced PDI was shown to disassemble the A1 subunit of cholera toxin and render it susceptible to proteolysis, while upon oxidation with GSSG, PDI was no longer able to perform this task. This was independent of any thiol/disulfide exchange reactions between PDI and the cholera toxin; rather, it was due to differences in PDI's protein conformation upon oxidation and reduction. While reduced PDI was able to bind and unfold the A1 chain of cholera toxin, oxidized PDI lost this high-affinity binding state and released the protein. Thus, the authors proposed that what ATP binding and hydrolysis does for nucleotide-dependent chaperones such as Hsp70, the redox conditions do for nucleotide-independent proteins such as PDI and Hsp33 [42] (fig. 2). Energy for the conformational changes might not any longer be provided by ATP hydrolysis but by the redox-driven formation and breakage of intramolecular disulfide bonds.

\section{Redox regulation: an emerging field}

Hsp33 and PDI are two members of a rapidly expanding family of redox-regulated proteins. Many of those members contain cysteine centers that are involved in zinc coordination, making them some of the strongest nucleophiles in the cell and prime targets for fast oxidation [43]. These include metallothionein [16, 17], the disulfide stress anti-sigma factor of Streptomyces coelicolor RsrA [44, 45], several zinc-finger proteins and transcription factors [46, 47], and protein kinase C [48]. Targets for redox regulation are not limited to proteins with zinc centers, however. Both the eukaryotic oxidative stress transcription factor Yap1 [49] and the prokaryotic oxidative stress transcription factor OxyR [10] sense oxidative stress in the cytosol by means of a single disulfide bond [12]. Another mode of redox sensing has been elucidated for the anaerobic global transcription regulator of $E$. coli, FNR; it uses an $[4 \mathrm{Fe}-4 \mathrm{~S}]^{2+}$ cluster for sensing oxygen 


\section{low affinity binding state}
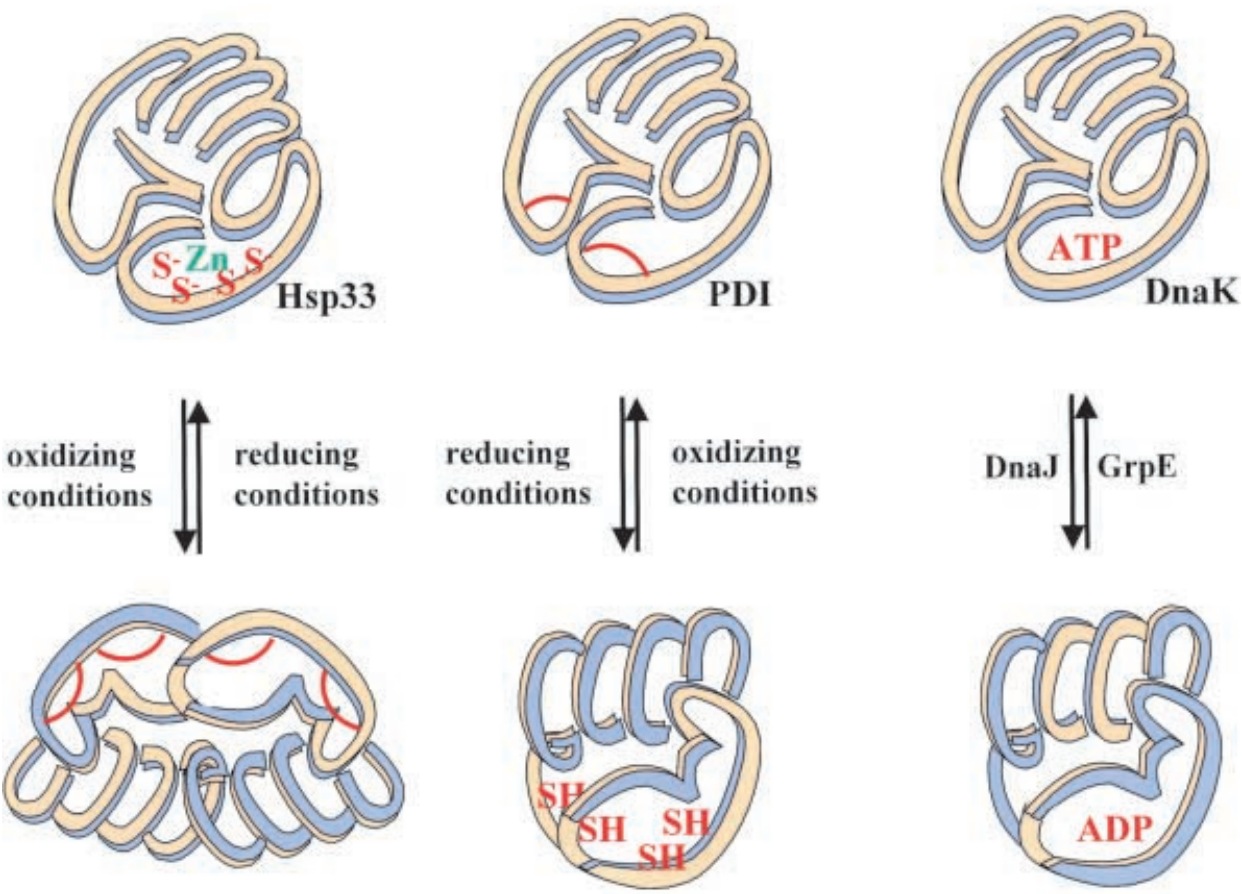

\section{high affinity binding state}

Figure 2. Three different ways to regulate chaperone activity. Molecular chaperones cycle between low-affinity and high-affinity substrate binding states. Redox-dependent chaperones such as Hsp33 or PDI use reversible disulfide bond formation for their regulation, while DnaK is a nucleotide-dependent chaperone that uses ATP binding and hydrolysis for regulation [42]. In the case of DnaK, the action of co-chaperones is required that accelerates ATP hydrolysis (DnaJ) and facilitates the ATP for ADP exchange reaction (GrpE). Disulfide bonds in Hsp33 and PDI are shown as red lines.

[50]. Upon exposure of FNR to oxygen, the $[4 \mathrm{Fe}-4 \mathrm{~S}]^{2+}$ in FNR converts into a $[2 \mathrm{Fe}-2 \mathrm{~S}]^{2+}$ cluster, a process that causes the reversible inactivation of the transcription factor [51].

Common to all these redox-regulated proteins is their ability to sense changes in the redox conditions of their environment and to quickly translate them into changes in protein conformation and, more important, activity. Given that oxidative stress not only accompanies heat shock but is also implicated in many other pathological situations such as cancer [52] and aging [53], it will be interesting to determine how many other proteins use this very elegant mode of functional regulation.

\section{Conclusion}

The concept of regulating the affinity of chaperones via changes in protein conformation has been observed many times before, by means of ATP binding and hydrolysis, as is the case GroEL and DnaK [54], or by means of changing the oligomerization state, as has been shown for the ATP-independent small Hsps, Hsp26 and Hsp18.1 [55,
56]. Hsp33 and PDI have now been shown to use a novel concept of regulation where the redox conditions of the environment power a disulfide switch within the proteins $[5,42]$. The formation or breakage of disulfide bonds causes conformational changes in the proteins that lead to either high or low substrate binding affinity. In the case of PDI, simple disulfide bond formation is sufficient for this process. Hsp33's regulation of its chaperone activity appears to have reached a new level of sophistication; activation of Hsp33 requires the combined effort of disulfide bond formation, zinc release and dimerization.

Acknowledgement. We thank Dr James Bardwell and Jörg Hoffmann for numerous discussions and for critically reading the manuscript. This work was supported by a Burroughs Wellcome Fund Career Award and a Biological Scholar Award from the University of Michigan to U.J.

1 Nakai K. and Horton P. (1999) PSORT: a program for detecting sorting signals in proteins and predicting their subcellular localization. Trends Biochem. Sci. 24: 34-36

2 Chuang S. E. and Blattner F. R. (1993) Characterization of twenty-six new heat shock genes of Escherichia coli. J. Bacteriol. 175: $5242-5252$ 
3 Korber P., Stahl J. M., Nierhaus K. H. and Bardwell J. C. A. (2000) Hsp15: a ribosome-associated heat shock protein. EMBO J. 19: $741-748$

4 Richmond C. S., Glasner J. D., Mau R., Jin H. and Blattner F. R. (1999) Genome-wide expression profiling in Escherichia coli K-12. Nucleic Acids Res. 27: 3821-3835

5 Jakob U., Muse W., Eser M. and Bardwell J. C. A. (1999) Chaperone activity with a redox switch. Cell 96: $341-352$

6 Lorimer G. H. (1996) A quantitative assessment of the role of the chaperonin proteins in protein folding in vivo. FASEB J. 10: $5-9$

7 Gilbert H. F. (1990) Molecular and cellular aspects of thioldisulfide exchange. Advances in Enzymology and Related Areas of Molecular Biology 63: 69-172

8 Ruddock L. W. and Klappa P. (1999) Oxidative stress: protein folding with a novel redox switch. Curr. Biol. 9: R400-402

9 Aslund F. and Beckwith J. (1999) Bridge over troubled waters: sensing stress by disulfide bond formation. Cell 96: 751-753

10 Zheng M., Aslund F. and Storz G. (1998) Activation of the OxyR transcription factor by reversible disulfide bond formation. Science 279: $1718-1721$

11 Aslund F., Zheng M., Beckwith J. and Storz G. (1999) Regulation of the OxyR transcription factor by hydrogen peroxide and the cellular thiol-disulfide status. Proc. Natl. Acad. Sci. USA 96: $6161-6165$

12 Choi H., Kim S., Mukhopadhyay P., Cho S., Woo J., Storz G. et al. (2001) Structural basis of the redox switch in the OxyR transcription factor. Cell 105: $103-113$

13 Zheng M., Wang X., Templeton L. J., Smulski D. R., LaRossa R. A. and Storz G. (2001) DNA microarray-mediated transcriptional profiling of the Escherichia coli response to hydrogen peroxide. J. Bacteriol. 183: 4562-4570

14 Zheng M., Wang X., Doan B., Lewis K. A., Schneider T. D. and Storz G. (2001) Computation-directed identification of OxyR DNA binding sites in Escherichia coli. J. Bacteriol. 183: 4571-4579

15 Jakob U., Eser M. and Bardwell J. C. A. (2000) Redox switch of hsp33 has a novel zinc-binding motif. J. Biol. Chem. 275: $38302-38310$

16 Jacob C., Maret W. and Vallee B. L. (1998) Control of zinc transfer between thionein, metallothionein, and zinc proteins. Proc. Natl. Acad. Sci. USA 95: 3489-3494

17 Maret W. and Vallee B. L. (1998) Thiolate ligands in metallothionein confer redox activity on zinc clusters. Proc. Natl. Acad. Sci. USA 95: $3478-3482$

18 Barbirz S., Jakob U. and Glocker M. O. (2000) Mass spectrometry unravels disulfide bond formation as the mechanism that activates a molecular chaperone. J. Biol. Chem. 275: 18759-18766

19 Graumann J., Lilie H., Tang X., Tucker K. A., Hoffmann J. H., Vijayalakshmi J. et al. (2001) Activation of the redox-regulated molecular chaperone Hsp33 - a two-step mechanism. Structure 9: $377-387$

20 Hightower K. E. and Fierke C. A. (1999) Zinc-catalyzed sulfur alkyation: insights from protein farnesyltransferase. Curr. Opin. Chem. Biol. 3: 176-181

21 Kim S. J., Jeong D. G., Chi S. W., Lee J. S. and Ryu S. E. (2001) Crystal structure of proteolytic fragments of the redox-sensitive Hsp33 with constitutive chaperone activity. Nat. Struct. Biol. 8: 459-466

22 Raman B., Siva Kumar L. V., Ramakrishna T. and Mohan Rao Ch. (2001) Redox-regulated chaperone function and conformational changes of Escherichia coli Hsp33. FEBS Lett. 489: 19-24

23 Jakob U., Lilie H., Meyer I. and Buchner J. (1995) Transient interaction of $\mathrm{Hsp} 90$ with early unfolding intermediates of citrate synthase. Implications for heat shock in vivo. J. Biol. Chem. 270: 7288-7294

24 Buchner J., Grallert H. and Jakob U. (1998) Analysis of chaperone function using citrate synthase as nonnative substrate protein. Methods Enzymol. 290: 323-338
25 Vijayalakshmi J., Mukhergee M. K., Graumann J., Jakob U. and Saper M. A. (2001) The 2.2 A crystal structure of Hsp33: a heat shock protein with redox- regulated chaperone activity. Structure 9: $367-375$

26 Storz G. and Imlay J. A. (1999) Oxidative stress. Curr. Opin. Microbiol. 2: 188-194

27 Rietsch A. and Beckwith J. (1998) The genetics of disulfide bond metabolism. Annu. Rev. Genet. 32: 163-184

28 Freeman M. L., Spitz D. R. and Meredith M. J. (1990) Does heat shock enhance oxidative stress? Studies with ferrous and ferric iron. Radiat. Res. 124: 288-293

29 Hassett D. J. and Cohen M. S. (1989) Bacterial adaptation to oxidative stress: implications for pathogenesis and interaction with phagocytic cells. FASEB J. 3: 2574-2582

30 Miller R. and Britigan B. (1997) Role of oxidants in microbial pathophysiology. Clin. Microbiol. Rev. 10: $1-18$

31 Halliwell B. and Gutteridge J. M. (1984) Oxygen toxicity, oxygen radicals, transition metals and disease. Biochem. J. 219: $1-14$

32 McDuffee A. T., Senisterra G., Huntley S., Lepock J. R., Sekhar K. R., Meredith M. J. et al. (1997) Proteins containing non-native disulfide bonds generated by oxidative stress can act as signals for the induction of the heat shock response. J. Cell. Physiol. 171: $143-151$

33 Spitz D. R., Dewey W. C. and Li G. C. (1987) Hydrogen peroxide or heat shock induces resistance to hydrogen peroxide in Chinese hamster fibroblasts. J. Cell. Physiol. 131: 364-373

34 Davidson J. F., Whyte B., Bissinger P. H. and Schiestl R. H. (1996) Oxidative stress is involved in heat-induced cell death in Saccharomyces cerevisiae. Proc. Natl. Acad. Sci. USA 93: $5116-5121$

35 Benov L. and Fridovich I. (1995) Superoxide dismutase protects against aerobic heat shock in Escherichia coli. J. Bacteriol. 177: 3344-3346

36 Davidson J. F. and Schiestl R. H. (2001) Cytotoxic and genotoxic consequences of heat stress are dependent on the presence of oxygen in Saccharomyces cerevisiae. J. Bacteriol. 183: $4580-4587$

37 VanBogelen R. A., Kelley P. M. and Neidhardt F. C. (1987) Differential induction of heat shock, SOS, and oxidation stress regulons and accumulation of nucleotides in Escherichia coli. J. Bacteriol. 169: 26-32

38 Marini M., Frabetti F., Musiani D. and Franceschi C. (1996) Oxygen radicals induce stress proteins and tolerance to oxidative stress in human lymphocytes. Int. J. Radiat. Biol. 70: $337-350$

39 Wheeler J. C., Bieschke E. T. and Tower J. (1995) Muscle-specific expression of Drosophila hsp70 in response to aging and oxidative stress. Proc. Natl. Acad. Sci. USA 92: 10408-10412

40 Stadtman E. R. (1990) Metal ion-catalyzed oxidation of proteins: biochemical mechanism and biological consequences. Free Radic. Biol. Med. 9: 315-325

41 Noiva R. (1999) Protein disulfide isomerase: the multifunctional redox chaperone of the endoplasmic reticulum. Seminars in Cell and Developmental Biology 10: 481-493

42 Tsai B., Rodighiero C., Lencer W. I. and Rapoport T. A. (2001) Protein disulfide isomerase acts as a redox-dependent chaperone to unfold cholera toxin. Cell 104: 937-948

43 Cappel R. E. and Gilbert H. F. (1988) Thiol/disulfide exchange between 3-hydroxy-3-methylglutaryl-CoA reductase and glutathione. A thermodynamically facile dithiol oxidation. J. Biol. Chem. 263: 12204-12212

44 Kang J. G., Paget M. S., Seok Y. J., Hahn M. Y., Bae J. B., Hahn J. S. et al. (1999) RsrA, an anti-sigma factor regulated by redox change. EMBO J. 18: 4292-4298

45 Paget M. S., Bae J. B., Hahn M. Y., Li W., Kleanthous C., Roe J. H. et al. (2001) Mutational analysis of RsrA, a zinc-binding anti-sigma factor with a thiol-disulphide redox switch. Mol. Microbiol. 39: 1036-1047 
46 Wu X., Bishopric N. H., Discher D. J., Murphy B. J. and Webster K. A. (1996) Physical and functional sensitivity of zinc finger transcription factors to redox change. Mol. Cell. Biol. 16: $1035-1046$

47 Park J. S., Wang M., Park S. J. and Lee S. H. (1999) Zinc finger of replication protein A, a non-DNA binding element, regulates its DNA binding activity through redox. J. Biol. Chem. 274: $29075-29080$

48 Knapp L. T. and Klann E. (2000) Superoxide-induced stimulation of protein kinase $\mathrm{C}$ via thiol modification and modulation of zinc content. J. Biol. Chem. 275: 24136-24145

49 Delaunay A., Isnard A. D. and Toledano M. B. (2000) $\mathrm{H}_{2} \mathrm{O}_{2}$ sensing through oxidation of the Yap1 transcription factor. EMBO J. 19: 5157-5166

50 Khoroshilova N., Beinert H. and Kiley P. J. (1995) Association of a polynuclear iron-sulfur center with a mutant FNR protein enhances DNA binding. Proc. Natl. Acad. Sci. USA 92: 2499-2503

51 Kiley P. J. and Beinert H. (1999) Oxygen sensing by the global regulator, FNR: the role of the iron- sulfur cluster. FEMS Microbiol. Rev. 22: 341-352
52 Kaneko T., Yamashima T., Tohma Y., Nomura M., Imajoh-Ohm S., Saido T. C. et al. (2001) Calpain-dependent proteolysis of merlin occurs by oxidative stress in meningiomas: a novel hypothesis of tumorigenesis. Cancer 92: 2662-2672

53 Squier T. C. (2001) Oxidative stress and protein aggregation during biological aging. Exp. Gerontol. 36: 1539-1550

54 Bukau B. and Horwich A. L. (1998) The Hsp70 and Hsp60 chaperone machines. Cell 92: 351-366

55 Haslbeck M., Walke S., Stromer T., Ehrnsperger M., White H. E., Chen S. et al. (1999) Hsp26: a temperature-regulated chaperone. EMBO J. 18: 6744-6751

56 Lee G. J., Roseman A. M., Saibil H. R. and Vierling E. (1997) A small heat shock protein stably binds heat-denatured model substrates and can maintain a substrate in a folding-competent state. EMBO J. 16: 659-671

57 Reprinted from Structure, Vol. 9, Graumann J., Lilie H., Tang X., Tucker K. T., Hoffmann J. H., Vijayalakshmi J. et al. Activation of the Redox regulated molecular chaperone Hsp33-A two-step mechanism, pp. 377-387, Copyright (2001), with permission from Elsevier Science.

\section{(2) To access this journal online: \\ (20) http://www.birkhauser.ch}

